\title{
Level Occupancy Anomalies in a Double QD System
}

\author{
P. STEFAŃSKI \\ Institute of Molecular Physics, Polish Academy of Sciences \\ Smoluchowskiego 17, 60-179 Poznań, Poland
}

\begin{abstract}
A double quantum dot system is considered in the presence of the electron intra-Coulomb $U$ interactions within the dots and inter-Coulomb $U_{12}$ interactions between them. We show that both for spinless and spinfull electrons the dots occupancy anomalies are encountered and caused by electron correlations. This non-monotonic behavior strongly deviates from usual level filling at the Coulomb blockade. For spinfull electrons the inter-dot interaction modifies also on-site electron correlations.
\end{abstract}

PACS numbers: 73.63.Kv, 73.23.Hk

\section{Introduction}

The occupancy anomalies encountered in quantum dot systems are interesting phenomena for various aspects. They can be caused by two generic for nanodevices phenomena: quantum interference and/or electron-electron correlations. These non-monotonicities are considered as a cause of the so-called "phase lapse" [1] by the unusual modification of the Friedel sum rule and phase evolution of the electron wave propagating through the dot in the Aharonov-Bohm geometry. They can also be bound to intriguing phenomenon called " 0.7 anomaly" [2] in short quantum point contacts, in which the occupation of the first subband is also modified by interactions. When polarized leads are considered, the quantum dot (QD) occupancy anomalies lead to an idea of the spin polarizer fully controlled electrically [3].

Until recently the occupancy anomalies have been studied mainly for spinless electrons [4]. We extend the considerations to the spinfull case exhibiting more direct correspondence with experimental situation.

\section{Hamiltonian of the system}

A system composed of two quantum dots in the Coulomb blockade regime, coupled to the leads is considered. Each dot is assumed to have one spin-degenerate active level indexed with $\gamma=1,2$. An inter-level Coulomb interaction is also assumed between them with the strength of $U_{12}$. The Hamiltonian describing the system is: $H=H_{\mathrm{QDs}}+H_{\text {leads }}+H_{\text {int }}$, where

$$
\begin{aligned}
& H_{\mathrm{QDs}}=\sum_{\gamma=1,2}\left[\sum_{\sigma} \epsilon_{\gamma} d_{\gamma \sigma}^{+} d_{\gamma \sigma}+\sum_{k, \sigma}\left(t_{\gamma} c_{k \sigma}^{+} d_{\gamma \sigma}+\text { h.c. }\right)\right], \\
& H_{\text {leads }}=\sum_{k, \sigma} \epsilon_{k} c_{k \sigma}^{+} c_{k \sigma},
\end{aligned}
$$

$$
H_{\mathrm{int}}=\sum_{\gamma=1,2} U_{\gamma} n_{\gamma \sigma} n_{\gamma \bar{\sigma}}+\sum_{\sigma} U_{12} n_{1 \sigma} n_{2 \bar{\sigma}}
$$

The dots levels are coupled to the separate leads (the index numbering the leads is suppressed for brevity) by the matrix elements $t_{\gamma}$ and get finite width of $\Gamma_{\gamma \sigma}=$ $\pi\left|t_{\gamma}\right|^{2} \rho_{\sigma}$. The $\rho_{\sigma}$ is the leads density of states assumed to be featureless and broad. Both the QDs levels are uniformly shifted with respect to the Fermi level, $\epsilon_{\mathrm{F}}=0$, by capacitative action of gate voltage: $\epsilon_{\gamma} \equiv \epsilon_{\gamma}-V_{\mathrm{g}}$. The Hamiltonian $H_{\text {int }}$ describes intra- $U_{\gamma}$ and inter- $U_{12}$ Coulomb interactions in the system.

The discussed system is the simplest one for considering the charge and spin dynamics inside the dots in presence of on-site and inter-site interactions. When a finite splitting between the levels is present, it can also be considered as a system describing single two-level quantum dot.

\section{Spinless electrons}

Limit of spinless electrons can be realized by applying large external magnetic field which introduces Zeeman splitting of each dot level. The probability of the occupancy of the higher in energy spin sub-level is very small leaving only the lower sub-level occupied. In such a situation the on-site Coulomb interactions $U_{\gamma}=0$ and the only interaction left is between the dots levels governed by $U_{12}$.

For the limiting degenerate case of $\epsilon_{1}=\epsilon_{2}$ the spinless Hamiltonian is formally equivalent to the single spin-degenerate impurity Anderson model [5]. Thus, the Hubbard approximation [6] can be adopted for the calculation of quantum dots Green's function with the correspondence $\sigma \rightarrow \gamma$ and $\bar{\sigma} \rightarrow \gamma^{\prime}$. The Hubbard approximation is the most suitable for electron correlations description in the Coulomb blockade regime, $U \gg \Gamma$. The Fourier transformed expression for retarded $\gamma$-level 
Green's function $G_{\gamma}^{\mathrm{r}}\left(t-t^{\prime}\right)=-\mathrm{i} \theta\left(t-t^{\prime}\right)\left\langle\left[d_{\gamma}(t) d_{\gamma}^{\dagger}\left(t^{\prime}\right)\right]_{+}\right\rangle \equiv$ $\left\langle\left\langle d_{\gamma}, d_{\gamma}^{\dagger}\right\rangle\right\rangle$ has the form

$$
\begin{aligned}
& G_{\gamma}^{\mathrm{r}}(\omega)=\left(\frac{\omega-\epsilon_{\gamma}}{1+\frac{\left\langle n_{\gamma^{\prime}} U_{12}\right.}{\omega-\epsilon_{\gamma}-U_{12}}}+\mathrm{i} \Gamma_{\gamma}\right)^{-1} \simeq \frac{1-\left\langle n_{\gamma^{\prime}}\right\rangle}{\omega-\epsilon_{\gamma}+\mathrm{i} \Gamma_{\gamma}} \\
& +\frac{\left\langle n_{\gamma^{\prime}}\right\rangle}{\omega-\epsilon_{\gamma}-U_{12}+\mathrm{i} \Gamma_{\gamma}} .
\end{aligned}
$$

The dots Green functions have the form of two Hubbard peaks centered at $\omega=\epsilon_{\gamma}$ and $\omega=\epsilon_{\gamma}+U_{12}$. The weight of the spectral density peaks of the $\epsilon_{\gamma}$ level is governed by the occupancy of the other $\gamma^{\prime}$ level and vice versa. It causes anomalies of the level occupancies for $\epsilon_{1} \neq \epsilon_{2}$ or/and when $\Gamma_{1} \neq \Gamma_{2}$ because it immediately implies the occupancy difference of $\left\langle n_{\gamma}\right\rangle$ and $\left\langle n_{\gamma^{\prime}}\right\rangle$. In the present paper we concentrate on the case of $\Gamma_{1} \neq \Gamma_{2}$ and $\epsilon_{1}=\epsilon_{2}$. The level occupancies have been calculated self-consistently for a given gate voltage and level width from the set of coupled integral equations involving appropriate QDs spectral densities $\rho_{\gamma}(\omega)=-\frac{1}{\pi} \Im G_{\gamma}^{\mathrm{r}}(\omega)$ (in $T=0)$ :

$$
\begin{aligned}
& \left\langle n_{\gamma}\right\rangle=\int_{-\infty}^{\epsilon_{\mathrm{F}}} \rho_{\gamma}\left(\omega,\left\langle n_{\gamma^{\prime}}\right\rangle\right) \mathrm{d} \omega, \\
& \left\langle n_{\gamma^{\prime}}\right\rangle=\int_{-\infty}^{\epsilon_{\mathrm{F}}} \rho_{\gamma^{\prime}}\left(\omega,\left\langle n_{\gamma}\right\rangle\right) \mathrm{d} \omega .
\end{aligned}
$$

They are shown as well as corresponding correlators in Fig. 1 for various ratios of $\Gamma_{2} / \Gamma_{1}$. For $\Gamma_{2} / \Gamma_{1}=1$ the model coincides with single impurity Anderson model with on-site Coulomb interaction $U_{12}$. In this case both the occupancies $\left\langle n_{1}\right\rangle$ and $\left\langle n_{2}\right\rangle$ shown in parts (a) and (c) have usual $V_{\mathrm{g}}$ dependence of the Coulomb-blockaded level with plateau $\sim U_{12}$. When $\Gamma_{2} \neq \Gamma_{1}$, the non-monotonicity in the level occupancies develops. The maxima of $\left\langle n_{1}\right\rangle$ correspond to minima of $\left\langle n_{2}\right\rangle$ and vice versa. There are also three degeneracy points where $\left\langle n_{1}\right\rangle=\left\langle n_{2}\right\rangle$. Two of them for $V_{\mathrm{g}}=0$ and $V_{\mathrm{g}}=U_{12}$ correspond to situation when the Hubbard dots levels $\epsilon_{\gamma}$ and $\epsilon_{\gamma}+U_{12}(\gamma=1,2)$ cross Fermi energy, respectively. The third point is at the occupancy plateau $n_{\gamma} \approx 0.5$, when the $\epsilon_{\mathrm{F}}$ is placed in-between QDs Hubbard levels.

The inter-correlation functions, $\left\langle n_{\gamma^{\prime}} n_{\gamma}\right\rangle$, are calculated from the spectral representation of appropriate two-particle Green's functions $\left\langle\left\langle n_{\gamma^{\prime}} d_{\gamma}, d_{\gamma}^{\dagger}\right\rangle\right\rangle$ obtained in the Hubbard approximation. The correlation measure between electrons on $\gamma$ and $\gamma^{\prime}$ levels is described by appropriate correlator: $C_{\gamma}=\left\langle n_{\gamma^{\prime}} n_{\gamma}\right\rangle-\left\langle n_{\gamma^{\prime}}\right\rangle\left\langle n_{\gamma}\right\rangle$ shown in parts (b) and (d) of Fig. 1. It can be noted that: (i) the maximum of electron-electron correlations takes place for symmetric Hubbard levels arrangement for $\epsilon_{\gamma}=$ $-U_{12} / 2\left(=-V_{\mathrm{g}}\right)$. The correlators reach minimum at this point because correlated motion of electrons sufficiently reduces the Coulomb repulsion between them. For $\Gamma_{2}=\Gamma_{1}, C_{1}=C_{2}$ and can be regarded as onsite correlations on spin-degenerate level. (ii) The correlations on $\epsilon_{2}$ increase with the decrease in $\epsilon_{2}$ width. (iii) The correlations on $\epsilon_{1}$ are only slightly affected by
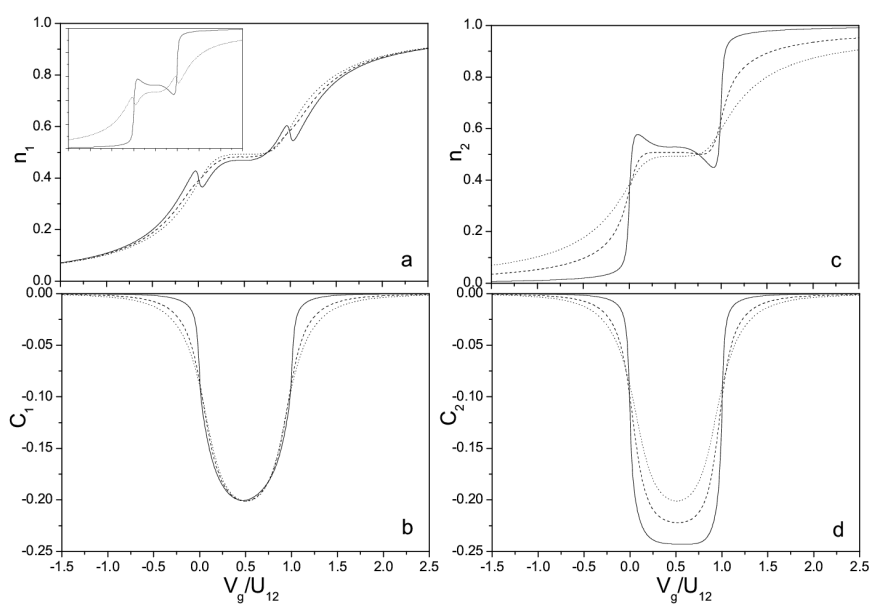

Fig. 1. Occupancies $\left\langle n_{1}\right\rangle,\left\langle n_{2}\right\rangle$ and correlators $C_{1}, C_{2}$ vs. gate voltage for $\Gamma_{1}=0.4 U_{12}$ and various ratios of $\Gamma_{2} / \Gamma_{1}: \Gamma_{2} / \Gamma_{1}=1-$ dotted curves, $\Gamma_{2} / \Gamma_{1}=0.5-$ dashed curves and $\Gamma_{2} / \Gamma_{1}=0.1-$ solid curves. Inset to part (a): the same occupancies $\left\langle n_{1}\right\rangle$-dotted curve and $\left\langle n_{2}\right\rangle$-solid curve for $\Gamma_{2} / \Gamma_{1}=0.1$ collected in one picture.

the change of $\Gamma_{2}$. (iv) The non-monotonicities in the $\left\langle n_{1}\right\rangle$ and $\left\langle n_{2}\right\rangle$ do not influence the correlations on the levels. An increase (decrease) in $\left\langle n_{1}\right\rangle$ occupancy is accompanied by corresponding decrease (increase) in $\left\langle n_{2}\right\rangle$ which compensates the possible changes of the correlated motion of electrons. Differently, for spinfull electrons, the on-site correlators are sensitive to inter-site Coulomb repulsion as shown below.

\section{Spinfull electrons}

For electrons with spin the full Hamiltonian, Eq. (1), is considered. There is no hopping between the dots which implies that $U_{12}$ is non-local and of capacitative nature. In contrast, $U_{\gamma}$ are of many-body, dynamical nature. Because of a different physical nature of intraand inter-Coulomb interactions a two-step procedure is applied to the Hamiltonian. In the first step, the $U_{12}$ as electrostatic interaction is treated in the Hartree-Fock (HF) approximation and the part of the $H_{\text {int }}$ Hamiltonian describing inter-Coulomb interactions takes the form: $\sum_{\sigma} U_{12}\left[\left\langle n_{1 \sigma}\right\rangle n_{2 \bar{\sigma}}+\left\langle n_{2 \bar{\sigma}}\right\rangle n_{1 \sigma}\right]$. It introduces a renormalization of the bare dot's levels, $\epsilon_{\gamma}^{\mathrm{HF}}=\epsilon_{\gamma}-V_{\mathrm{g}}+\left\langle n_{\gamma^{\prime} \sigma}\right\rangle U_{12}$ by the HF field acting between the dots. The effect is similar to the so-called "charge sensing" [7]. Thus, the effective Hamiltonian for the part describing the dots is

$$
\begin{aligned}
& H_{\mathrm{QDs}}^{\mathrm{eff}}=\sum_{\gamma}\left[\sum_{\sigma} \epsilon_{\gamma}^{\mathrm{HF}} d_{\gamma \sigma}^{+} d_{\gamma \sigma}+\sum_{k, \sigma}\left(t_{\gamma} c_{k \sigma}^{+} d_{\gamma \sigma}+\text { h.c. }\right)\right. \\
& \left.\quad+U_{\gamma} n_{\gamma \sigma} n_{\gamma \bar{\sigma}}\right] .
\end{aligned}
$$

The intra- $U_{\gamma}$ interactions are treated in the Hubbard approximation giving the dots Green functions of similar structure as in the spinless electron case, Eq. (2), but with the $\epsilon_{\gamma}$ dot level replaced by corresponding HF-level $\epsilon_{\gamma}^{\mathrm{HF}}$. 
Now, the self-consistently calculated occupancies of the $\gamma$ levels depend on the on-site occupancy of $\left\langle n_{\gamma \bar{\sigma}}\right\rangle$ via the Coulomb repulsion $U_{\gamma}$ and on the occupancy of the second $\operatorname{dot}\left\langle n_{\gamma^{\prime} \bar{\sigma}}\right\rangle$ via $U_{12}$ repulsion.

The correlation functions are calculated similarly as for spinless electrons but with effective Hamiltonian, Eq. (4). Now they describe intra-correlations between electrons of opposite spins of a given $\gamma$ level modified by HF field.

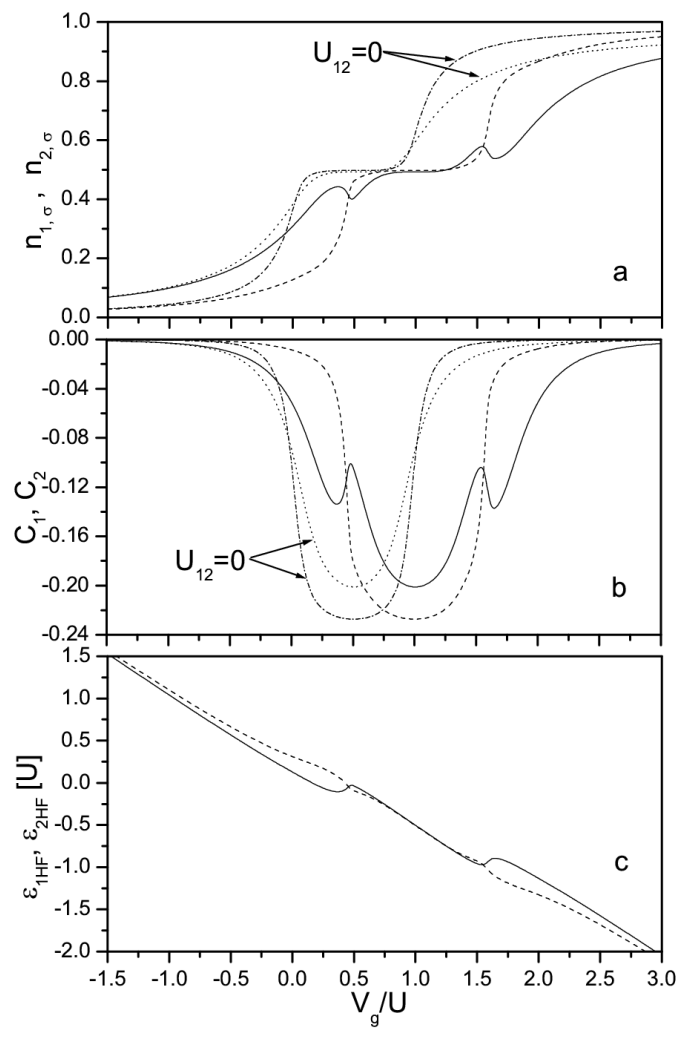

Fig. 2. (a) Occupancies $\left\langle n_{1 \sigma}\right\rangle$ (solid curve) and $\left\langle n_{2 \sigma}\right\rangle$ (dashed curve) vs. gate voltage calculated for $U_{1}=$ $U_{2}=U_{12}, \Gamma_{1 \sigma}=0.4 U, \Gamma_{2 \sigma}=0.4 \Gamma_{1 \sigma}$. The occupancies for $U_{12}=0$ are also displayed: $\left\langle n_{1 \sigma}\right\rangle$ - dotted, $\left\langle n_{2 \sigma}\right\rangle$ - dash-dotted curve. (b) Correlators calculated for the same parameters: $C_{1}$ - solid curve, $C_{2}$ - dashed curve. Correlators for $U_{12}=0$ : $C_{1}-$ dotted curve and $C_{2}$ dash-dotted curve. (c) Evolution of the Hartree-Fock levels: $\epsilon_{1 \mathrm{HF}}$ - solid and $\epsilon_{2 \mathrm{HF}}$ - dashed curve.

The results for $\Gamma_{2}=0.4 \Gamma_{1}$ and $U_{\gamma}=U_{12} \equiv U$ are collected in Fig. 2. Shown in part (a), the $\left\langle n_{1 \sigma}\right\rangle$ occupancy displays two anomalies being generated by rapidly changing Hartree-Fock field $\sim\left\langle n_{2 \bar{\sigma}}\right\rangle U_{12}$ acting on the bare $\epsilon_{1}$ level when the sharp, well-localized $\epsilon_{2}$ is shifted by gate voltage and populated by electrons. There are no such anomalies in $\left\langle n_{2 \sigma}\right\rangle$ curve because the field $\left\langle n_{1 \bar{\sigma}}\right\rangle U_{12}$ acting on $\epsilon_{2}$ changes smoothly for broader $\epsilon_{1}$. There are two degeneracy points at $V_{\mathrm{g}} \sim 0.5 U$ and $V_{\mathrm{g}} \sim 1.5 U$ where $\left\langle n_{1 \sigma}\right\rangle=\left\langle n_{2 \sigma}\right\rangle$. The HF fields acting on the dots levels are also equal $\left\langle n_{1 \bar{\sigma}}\right\rangle U_{12}=\left\langle n_{2 \bar{\sigma}}\right\rangle U_{12}$ at these points causing the Hartree-Fock level to cross (part (c)). At these points the electron correlation strength for both the dots is also equal; $C_{1}=C_{2}$ as shown in part (b). The overall shape of the correlators gets broader due to $U_{12}$ interaction. Both the plateaus are also shifted due to $\mathrm{HF}$ renormalization of the dots levels. The correlators have minimal value for $\epsilon_{\gamma}=-U / 2$, similarly as for spinless electrons. The sudden change of the occupancy of $\epsilon_{2}$ causes a decrease via $\mathrm{HF}$ field $\left\langle n_{2 \bar{\sigma}}\right\rangle U_{12}$ of the correlations on $\epsilon_{1}$ level. It is manifested by rapid jumps in the correlator $C_{1}$ at $V_{\mathrm{g}}$ of HF-level crossings. This effect can be opposed to the case of spinless electrons for which the increase in $\left\langle n_{\gamma}\right\rangle$ always was compensated by a decrease in $\left\langle n_{\gamma^{\prime}}\right\rangle$ and did not have influence of the correlation strength. The correlations on $\epsilon_{2}$ in turn are undisturbed due to smoothly changing $\left\langle n_{1 \bar{\sigma}}\right\rangle U_{12}$ field. All the on-site correlation anomalies are associated with the peculiar behavior of the HF-levels. Similarly as for spinless case, the strength of the on-site correlations is dependent on the level width and is larger for more localized $\epsilon_{2}$ level.

\section{Acknowledgments}

We acknowledge a valuable discussion with J. Morkowski. This work was supported as a part of European Science Foundation EUROCORES Programme FoNe by funds from the Ministry of Science and Higher Education and EC $6 \mathrm{FP}$ (contract N. ERAS-CT-2003-980409).

\section{References}

[1] M. Avinun-Kalish, M. Heiblum, O. Zarchin, D. Mahalu, V. Umansky, Nature 436, 529 (2005).

[2] S.M. Cronenwett, H.J. Lynch, D. GoldhaberGordon, L.P. Kouwenhoven, C.M. Marcus, K. Hirose, N.S. Wingreen, V. Umansky, Phys. Rev. Lett. 88, 226805 (2002).

[3] P. Stefański, Phys. Rev. B 77, 125331 (2008).

[4] J. König, Y. Gefen, Phys. Rev. B 71, 201308 (2005).

[5] P.W. Anderson, Phys. Rev. 124, 41 (1961).

[6] A.C. Hewson, Phys. Rev. 144, 420 (1966).

[7] P. Stefański, A. Tagliacozzo, B.R. Bułka, Solid State Commun. 125, 314 (2005). 